\title{
Occurrence of a lymphocele following renal transplantation
}

Allen $\underline{\operatorname{Sim}}^{1}$, MB BCh BAO, MRCS, Lay Guat $\underline{N g}^{1}$, MBBS, FRCS, Christopher $\underline{\text { Cheng }}{ }^{1}$, MBBS, FRCS

INTRODUCTION The incidence of lymphoceles - lymphatic collections around a transplanted kidney - can be as high as $20 \%$. We aimed to review the presentation, treatment and outcome of patients with lymphoceles.

METHODS We reviewed a prospective database of 154 patients who underwent renal transplantation at our hospital from January 2005 to November 2008.

RESULTS The mean age of the patients in our cohort was 46 (range 34-58) years. The incidence of lymphoceles in our series was $5.8 \%(n=9)$. The median onset was 19 (range $6-28)$ days post-transplantation, while the median size of the lymphoceles was 5 (range 1.5-8) cm. Lymphoceles were most commonly found at the lower pole of the transplanted kidney. Eight patients with lymphoceles had received cadaveric transplants. While a majority of these patients did not have hydronephrosis on presentation, four had markedly elevated creatinine. Of the nine patients with lymphoceles, six were on macrolides (tacrolimus, sirolimus or everolimus), two were successfully managed conservatively, three were managed percutaneously and four required surgical drainage via either laparoscopic marsupialisation $(n=1)$ or open drainage $(n=3)$. There was no graft loss.

CONCLUSION It remains unknown whether the choice of immunosuppressants increases the risk of lymphocele formation. Intervention is necessary in the case of impaired drainage of the pelvicalyceal system in these patients. Minimally invasive intervention, while effective in treating lymphoceles, does not provide definitive treatment. Surgical intervention should be considered early for the treatment of post-transplantation patients with lymphoceles, so as to shorten hospital stay and prevent further complications.

Keywords: Iymphocele, renal transplant

\section{INTRODUCTION}

A lymphocele is a lymphatic collection around the renal graft and urinary bladder. It can arise from either the lymph that drains through the lymphatic vessels in the sinus of the transplanted kidney or the lymphatic vessels surrounding the iliac vessels of the recipient. ${ }^{(1,2)}$ The reported incidence of lymphoceles ranges widely, from $1 \%-2 \%$ to as high as $20 \%{ }^{(1,2)}$ Factors associated with the aetiology of lymphoceles include acute rejection, length of surgery, extent of dissection and use of immunosuppressants. ${ }^{(1,2)}$ Small lymphoceles might be asymptomatic, but large ones can cause compression of the pelvicalyceal system, resulting in hydronephrosis and worsening renal function. ${ }^{(3)}$ The most common symptoms of lymphoceles are abdominal pain, lower limb oedema and urinary retention in the transplanted kidney, resulting in impaired graft function. Ultrasonography is the most common diagnostic tool used in the visualisation of lymphoceles. The aim of this study was to review the epidemiology, presentation, treatment and outcome of patients who underwent renal transplantation at our institute and subsequently developed lymphoceles.

\section{METHODS}

Between January 2005 and November 2008, 154 renal transplantations were performed at the Singapore General Hospital, Singapore. Although these procedures were performed by various surgeons at our institution, the surgical techniques used were the same. The renal graft was placed in the iliac fossa, vessels were anastomosed to the iliac vessels and the ureter was implanted into the bladder. All lymphatic channels of the recipient's iliac arteries and veins encountered during dissection were ligated with small titanium clips or diathermised, depending on the individual surgeon's preference. A double J stent was routinely inserted in the implanted ureter. A surgical drain was also inserted.

Baseline ultrasonography of the transplanted kidney was performed postoperatively and repeated whenever indicated. Patients with lymphoceles were managed conservatively if they were asymptomatic. However, intervention was considered necessary if the patient presented with hydronephrosis and worsening renal function. Intervention included percutaneous drainage of the collection, surgical drainage via laparoscopy, or open drainage. Percutaneous drainage was routinely performed under ultrasonographic guidance by an interventional radiologist. The lymphatic collection was aspirated until dry, and no sclerosant injections were used for any of the patients. In patients who underwent laparoscopic drainage, the omentum was inserted into the peritoneal window to prevent closure, whereas this was not necessary in those who underwent open surgical drainage, as the peritoneal window was large. The fluid aspirated from the collection was routinely sent for measurement of creatinine level to exclude the possibility of urinoma. 
Table I. Clinical presentations and use of immunosuppressants in the renal transplantation patients with lymphoceles $(n=9)$.

\begin{tabular}{|c|c|c|}
\hline Patient & Clinical presentation & Immunosuppressants \\
\hline 1 & Asymptomatic* & $\begin{array}{l}\text { Cyclosporine, mycophenolate } \\
\text { mofetil, prednisolone }\end{array}$ \\
\hline 2 & Raised creatinine & $\begin{array}{l}\text { Tacrolimus, myfortic, } \\
\text { prednisolone }\end{array}$ \\
\hline 3 & Asymptomatic* & $\begin{array}{l}\text { Azathioprine, prednisolone, } \\
\text { cyclosporine }\end{array}$ \\
\hline 4 & Raised creatinine & $\begin{array}{l}\text { Azathioprine, tacrolimus, } \\
\text { prednisolone }\end{array}$ \\
\hline 5 & Asymptomatic* & $\begin{array}{l}\text { Cyclosporine, myfortic, } \\
\text { prednisolone }\end{array}$ \\
\hline 6 & $\begin{array}{l}\text { Raised creatinine with } \\
\text { hydronephrosis }\end{array}$ & $\begin{array}{l}\text { Mycophenolate mofetil, } \\
\text { sirolimus, prednisolone }\end{array}$ \\
\hline 7 & Asymptomatic* & $\begin{array}{l}\text { Everolimus, cyclosporine, } \\
\text { prednisolone }\end{array}$ \\
\hline 8 & Asymptomatic* & $\begin{array}{l}\text { Everolimus, cyclosporine, } \\
\text { prednisolone }\end{array}$ \\
\hline 9 & $\begin{array}{l}\text { Raised creatinine with } \\
\text { hydronephrosis }\end{array}$ & $\begin{array}{l}\text { Sirolimus, mycophenolate } \\
\text { mofetil, prednisolone }\end{array}$ \\
\hline
\end{tabular}

${ }^{*}$ Routine ultrasonography was conducted in asymptomatic patients.

\section{RESULTS}

Out of the 154 patients who underwent renal transplantations, lymphoceles occurred in 9 (5.8\%) patients. The median onset of occurrence was 19 (range 6-28) days after surgery. The median age of patients diagnosed with lymphocele was 46 (range 34-58) years. The median operative time was 180 mins. The median maximum diameter of the lymphoceles was 5 (range 1.5-8.0) $\mathrm{cm}$. Diagnosis of lymphocele was made by ultrasonography, and no aspiration of the lymphocele was performed in the patients. The site of occurrence of lymphoceles included the inferior $(n=5)$, lateral $(n=2)$, upper $(n=1)$ and medial $(n=1)$ poles of the transplanted kidneys. Eight out of the nine patients $(88.9 \%)$ diagnosed with lymphocele had received cadaveric transplants, while $1(11.1 \%)$ patient received a living transplant from a relative. Out of these nine patients, 7 (77.8\%) had no hydronephrosis at presentation. Postoperatively, 2 (22.2\%) patients developed clinically significant hydronephrosis, 4 (44.4\%) had clinically elevated creatinine levels (Table I), and 6 $(66.7 \%)$ were on macrolides such as tacrolimus, sirolimus and everolimus. Although there were no delayed graft functions, there was one case of acute graft rejection.

Of the nine patients with lymphoceles, five were initially treated conservatively (Fig. 1). Among these five patients, three required subsequent intervention in the form of either percutaneous drainage or surgical intervention - two were managed successfully using percutaneous drainage, and one required repeated percutaneous drainage. The four patients who did not receive conservative treatment initially were treated with percutaneous drainage from the onset; however, due to failure of percutaneous drainage, two of these four patients had to undergo surgical treatment. In all, four patients were surgically treated because of failed percutaneous drainage, with drainage via the open method performed in three patients and

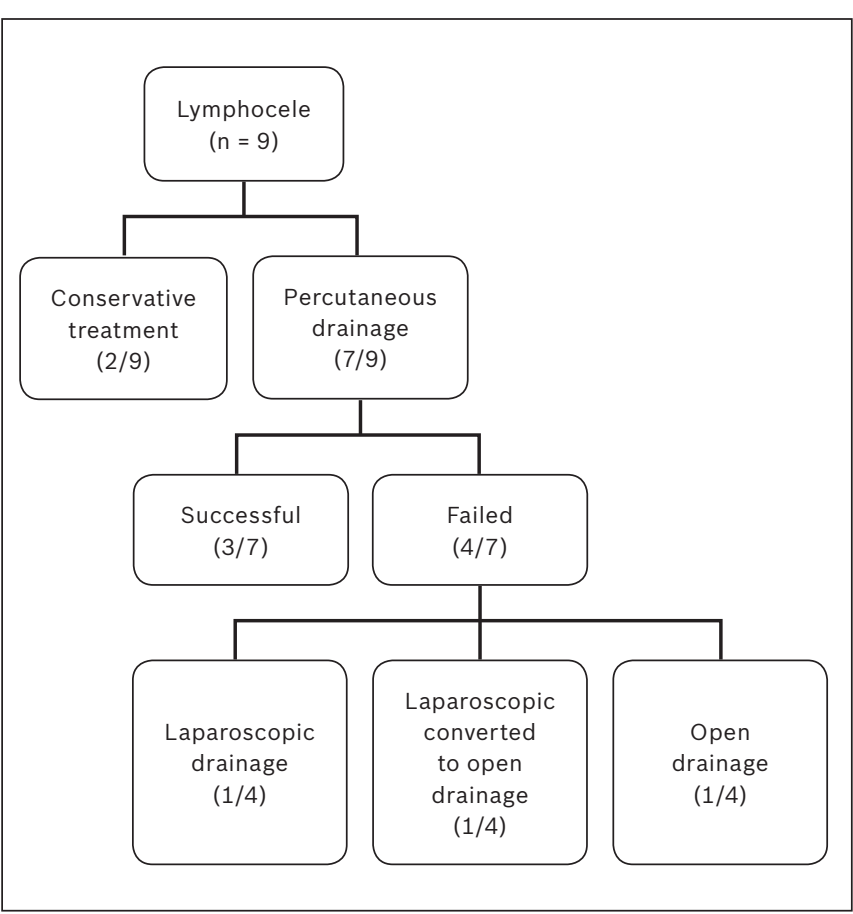

Fig. 1 Chart shows the various treatments received by patients with lymphoceles $(n=9)$.

laparoscopic drainage in one patient. Fluid culture was positive for Klebsiella spp. in only one out of seven patients who required intervention. Among the three patients who eventually underwent open drainage, two each had a sizeable lymphocele measuring $>4 \mathrm{~cm}$, and one had significant hydronephrosis and positive culture. In patients for whom conservative treatment failed, indications for intervention were an enlarging lymphocele size leading to worsening of renal function. There was no graft loss in any of the nine patients with lymphoceles. There was also no recurrence documented among any of the patients during the follow-up period (median 40 [range 38-84] months).

\section{DISCUSSION}

The reported incidence of symptomatic lymphoceles in the literature is $0.6 \%-18.0 \%$. ${ }^{(1)}$ The incidence of lymphoceles in our study was $5.8 \%(n=9)$, which is comparable to that reported in the literature. Although lymphoceles can be harmless and asymptomatic in many patients, they can also seriously affect renal function and may necessitate surgical intervention. Although asymptomatic lymphoceles do not require therapy, it is important that regular ultrasonographic monitoring continues until the collection is resolved. In the case of a large or symptomatic collection, therapy aims to efficiently and completely remove the collection. However, there is no gold standard of treatment for lymphoceles.

Percutaneous aspiration of the collection is associated with a high incidence $(50 \%-100 \%)$ of recurrence. ${ }^{(2)}$ Some authors have recommended laparoscopic marsupialisation as the firstline treatment of lymphoceles, ${ }^{(2,3)}$ while others have suggested initial percutaneous aspiration under ultrasonographic guidance, followed by sclerotherapy to prevent reaccumulation of fluid. $^{(4-6)}$ Percutaneous aspiration of lymphoceles has been shown 


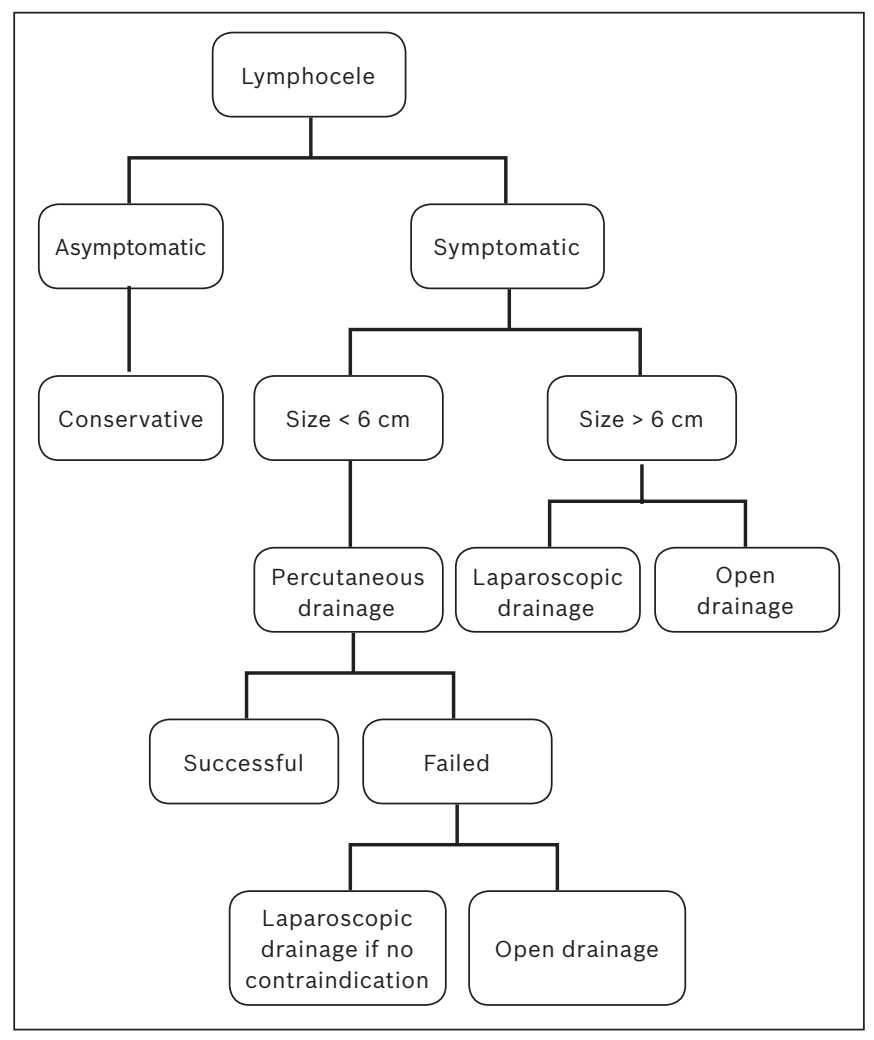

Fig. 2 Chart shows the management algorithm of patients with lymphoceles in the local setting.

to reduce the risk of recurrence (range $13 \%-33 \%),{ }^{(7-9)}$ as has additional sclerotherapy (range 6\%-25\%). ${ }^{(2,4,7)}$ Common sclerosing agents used in sclerotherapy are doxycycline and ethanol. ${ }^{(5)}$ Other sclerosing agents include povidone-iodine, talc and bleomycin. The use of sclerosing agents is generally considered safe and highly successful, although some reports have suggested that they have toxic effects on graft functions and may even cause graft loss. ${ }^{(10,11)}$ Surgical interventions for lymphoceles include marsupialisation, or external drainage via open or laparoscopic technique. The recurrence rates of lymphoceles following laparoscopic marsupialisation (range $6 \%-12 \%)^{(2,7)}$ and open drainage (range $6.7 \%-13 \%)^{(3,7)}$ are comparable. Laparoscopic marsupialisation is the recommended surgical intervention for lymphoceles, as it is less invasive, and has shorter operative time and hospital stay, as well as better cosmesis.

In our study, conservative and less invasive treatment, such as percutaneous drainage, was successful in $55.5 \%$ of patients. Among the four patients who had surgical intervention, one underwent laparoscopic marsupialisation and three underwent open drainage. Consistent with the results from other centres, ${ }^{(12)}$ three of these patients had larger sized lymphoceles $(>6 \mathrm{~cm}$ in diameter). We opted to perform open drainage in three patients under surgical intervention, as two of them had a history of previous abdominal surgery and one patient's collection was close to the hilum. There was no recurrence of lymphocele in any of our patients, regardless of the kind of treatment (percutaneous or surgical) used.

Although the surgical management of lymphoceles has remained largely unchanged, it remains unclear whether the introduction of newer immunosuppressants, such as tacrolimus, sirolimus and everolimus, has contributed to the increasing numbers of patients developing lymphoceles after renal transplantation. While studies have shown that sirolimus is a powerful immunosuppressant for renal transplant recipients, ${ }_{,}^{(13,14)}$ its use has been associated with a higher frequency of lymphocele formation. ${ }^{(13)}$ According to Srivastava et al, the incidence of lymphocele in patients treated with sirolimus is slightly higher than in those receiving mycophenolate mofetil. ${ }^{(15)}$ Even though the exact reasons for the development of lymphocele are still uncertain, avoiding the use of sirolimus in renal transplant recipients at the early postoperative period may help to reduce the incidence of lymphocele in these patients. Based on the results obtained from our local setting, we have developed an algorithm for the management of post-transplantation lymphoceles (Fig. 2).

The initial decision on whether a post-transplantation patient with lymphocele should be treated conservatively would depend on factors such as the size of the lymphocele, the symptoms observed, as well as the presence of hydronephrosis, impaired renal function or concomitant infection. If treatment is warranted, percutaneous drainage should be offered to patients. However, for patients with a collection $>6 \mathrm{~cm}$ in diameter, surgical options should be considered. In the event of a failed percutaneous intervention, surgical drainage should be offered to the patient. Laparoscopic drainage may also be offered, unless contraindicated due to small volume $(<100 \mathrm{~mL})$ of fluid, previous abdominal surgery, suspicion of infection, as well as location of lymphocele near the bladder, ureter or renal hilum.

In summary, the incidence of lymphocele was $5.8 \%$ in our series. Even though surgical techniques have remained unchanged for the most part, it is still unclear whether the introduction of newer immunosuppressants has contributed to the higher incidence of lymphoceles in post-renal transplantation patients. Intervention is required if the lymphocele causes an impairment in the drainage of the pelvicalyceal system. We found that although minimally invasive intervention was effective in treating lymphoceles, it did not provide a definitive treatment. Percutaneous drainage was found to be consistently associated with a higher recurrence rate, prolonged hospital stay, higher costs and more complications. Surgical intervention should be considered earlier in the treatment of patients with posttransplantation lymphoceles so as to shorten hospital stay and prevent further complications.

\section{REFERENCES}

1. Braun WE, Banowsky LH, Straffon RA, et al. Lymphocele associated with renal transplantation: Report of 15 cases and review of the literature. Am J Med 1974; 57:714-29.

2. Hsu TH, Gill IS, Grune MT, et al. Laparoscopic lymphocelectomy: a multiinstitutional analysis. J Urol 2000; 163:1096-8.

3. Fuller TF, Kang SM, Hirose R, et al. Laparoscopic treatment of symptomatic lymphocele after renal transplantation: laparoscopic versus open drainage. J Urol 2003; 169:2022-5.

4. Zuckerman DA, Yeager TD. Percutaeneous ethanol sclerotherapy of postoperative lymphoceles. AJR Am J Roentgenol 1997; 169:433-7. 
5. Hamza A, Fischer K, Koch E, et al. Diagnostic and therapy of lymphoceles after kidney transplantation. Transplant Proc 2006; 38:701-6.

6. Tasar M, Gulec B, Saglam M, et al. Posttransplant symptomatic lymphocele treatment with percutaneous drainage and ethanol sclerosis: long term follow up. Clin Imaging 2005; 29:109-16.

7. Atray NK, Moore F, Zaman F, et al. Post transplant lymphocele: a single centre experience. Clin Transplant 2004; 18 suppl 12:46-9.

8. Kim JK, Jeong YY, Kim YH, et al. Postoperative pelvic lymphocele: treatment with simple percutaneous catheter drainage. Radiology 1999; 212:390-4.

9. Bischof G, Rockenschaub S, Berlakovich G, et al. Management of lymphoceles after kidney transplantation. Transpl Int 1998; $11: 277-80$.

10. Manfro RC, Comerlato L, Berdichevski RH, et al. Nephrotoxic acute renal failure in a renal transplant patient with recurrent lymphocele treated with povidone-iodine irrigation. Am J Kidney Dis 2002; 40:655-7.
11. Adani GL, Baccarani U, Bresadola V, et al. Graft loss due to percutaneous sclerotherapy of a lymphocele using acetic acid after renal transplantation. Cardiovasc Intervent Radiol 2005; 28:836-8.

12. Krol R, Kolonko A, Chudek J, et al. Did volume of lymphocele after kidney transplantation determine the choice of treatment modality? Transplant Proc 2007; 39:2740-3.

13. Chiu MI, Katz H, Berlin V. RAPT1, a mammalian homolog of yeast TOR, interacts with the FKBP12/rapamycin complex. Proc Natl Acad Sci U S A 1994; 91:12574-8.

14. Kahan BD. Efficacy of sirolimus compared with azathioprine for reduction of acute renal allograft rejection: a randomised multicentre study. The Rapamune US Study Group. Lancet 2000; 356:194-202.

15. Srivastava A, Muruganandham K, Vinodh PB, et al. Post-renal transplant surgical complications with newer immunosuppressive drugs: mycophenolate mofetil vs. m-TOR inhibitors. Int Urol Nephrol 2010; 42:279-84.

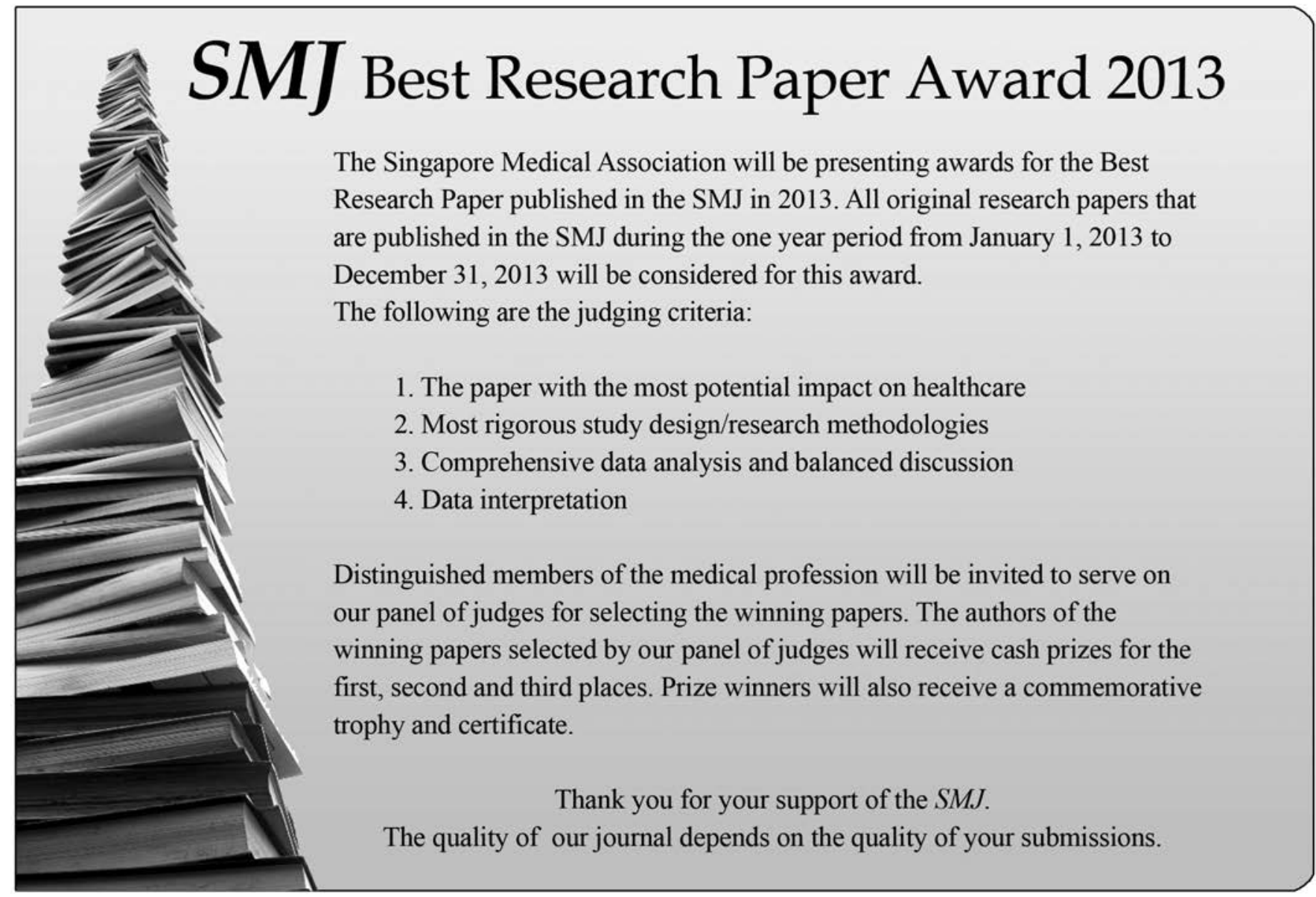

